\title{
Frontal Fibrosing Alopecia Associated with Lichen Planus Pigmentosus: Not Only in Dark Phototypes
}

\author{
María Eugenia Cappetta ${ }^{\mathrm{a}}$ Gabriela Álvarez $^{\mathrm{b}}$ Mónica Noguera ${ }^{\mathrm{a}}$ Gisela D’Atri ${ }^{\mathrm{c}}$ \\ Fernando Stengel $^{\mathrm{d}}$ \\ aDermatology, Instituto Universitario CEMIC, Buenos Aires, Argentina; ${ }^{\mathrm{b}}$ Dermatology, Instituto CIAL, \\ Bahía Blanca-Buenos Aires, Buenos Aires, Argentina; 'Dermatology, Grupo MCl, Buenos Aires, Argentina; \\ dDermatology, Buenos Aires Skin, Buenos Aires, Argentina
}

\section{Established Facts}

- Different associated facial clinical signs have been described within frontal fibrosing alopecia.

- The association of frontal fibrosing alopecia with lichen planus pigmetosus is mainly described in dark phototypes.

\section{Novel Insights}

- Frontal fibrosing alopecia and lichen planus pigmentosus can also occur in lower Fitzpatrick phototypes.

\section{Keywords}

Frontal fibrosing alopecia · Lichen planus pigmentosus

\section{Abstract}

The association between frontal fibrosing alopecia and lichen planus pigmetosus was first described in African women. Later, most reports about this association involved darkskinned patients. Here, we describe 5 cases of frontal fibrosing alopecia associated with lichen planus pigmentosus in light-skinned women from Argentina. Our communication highlights the strength of both entities' association also in lower Fitzpatrick phototypes.

(c) 2020 S. Karger AG, Basel

\section{Introduction}

Frontal fibrosing alopecia (FFA) is commonly described as a lichen planopilaris variant. It is not yet clear if these are distinct entities or belong to a spectrum of the same pathology [1]. FFA has a high aesthetic impact on patients, often affecting their quality of life severely. Since the first report in 1994 by Kossard [2], many series of patients have been published. It seems to be an epidemic type of alopecia, increasing in incidence in the last decades and mainly involving postmenopausal women. Because of the rise in its incidence, many environmental triggers had been postulated, but none had been proved as causal.
María Eugenia Cappetta

Dermatology, Instituto Universitario CEMIC 1234 Talcahuano St.

Buenos Aires C1014ADB (Argentina)

e_cappetta@hotmail.com 


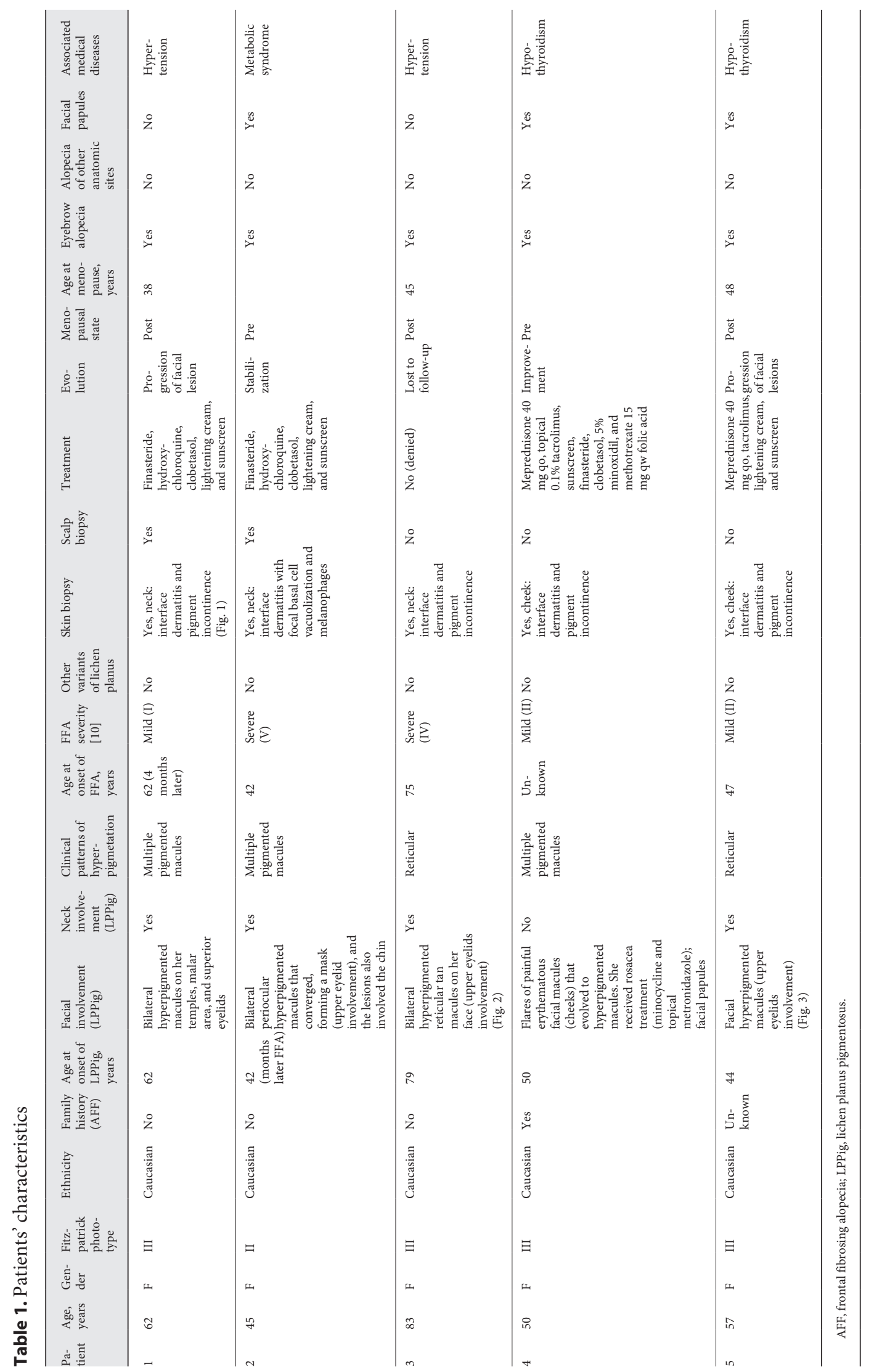




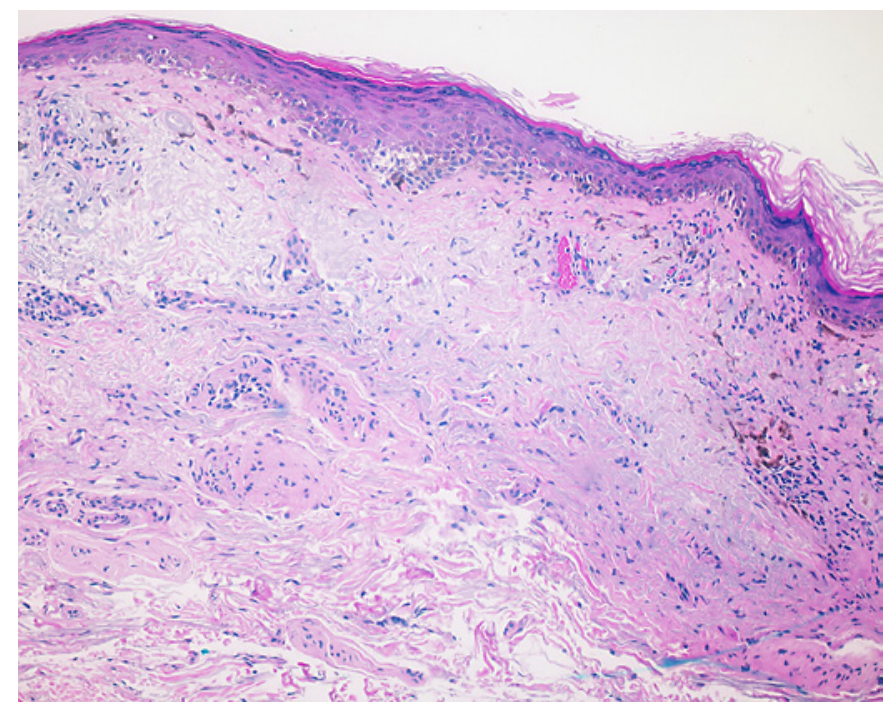

Fig. 1. Skin biopsy taken from the neck. $\mathrm{HE} \times 10$. Interface dermatitis and pigment incontinence. HE, hematoxylin and eosin.

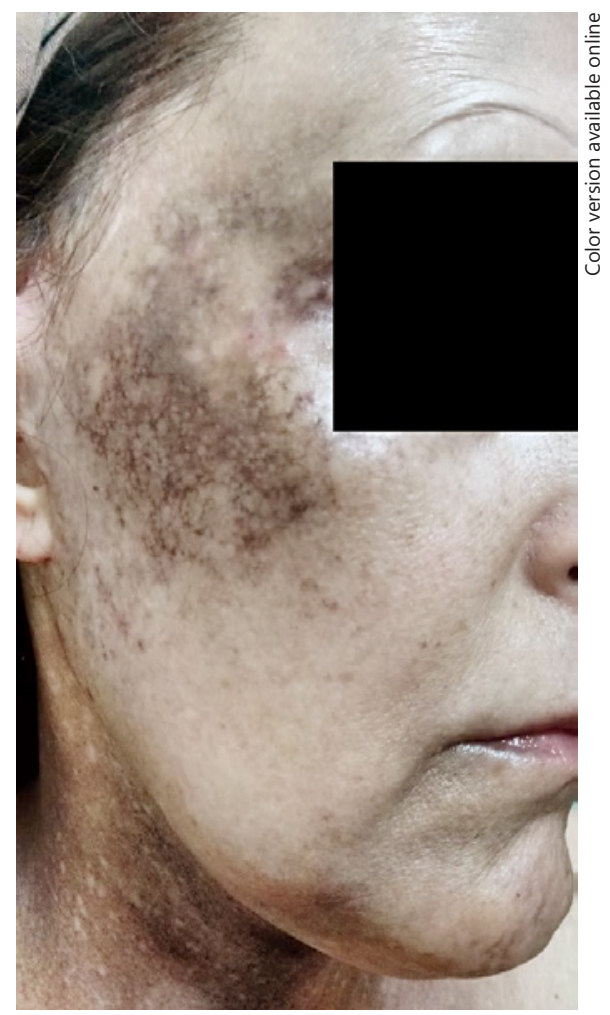

Fig. 3. Case 5: facial and neck hyperpigmented macules in a reticular distribution.

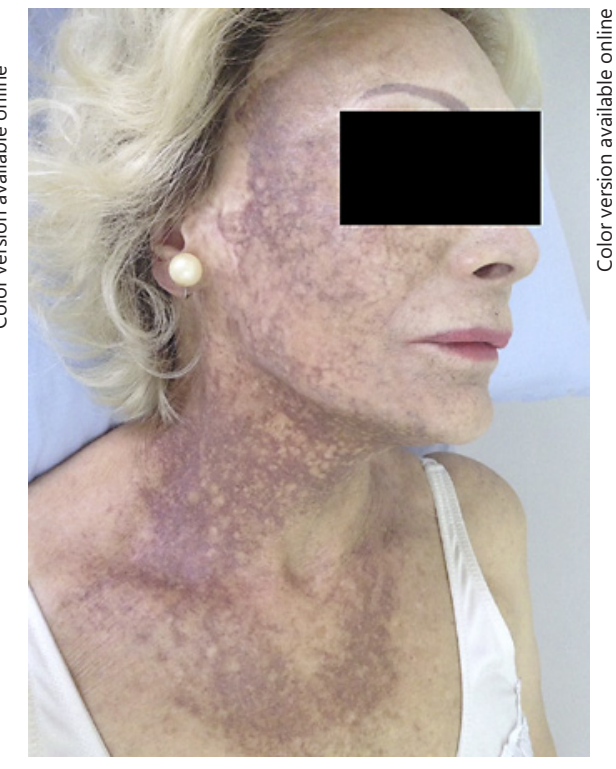

Fig. 2. Case 3: hyperpigmented reticular tan macules involving the face and neck.

Different associated clinical signs have been described, including depression of the frontal veins [3] and facial papules [4], among others. Within the lichenoid spectrum, associations with cutaneous lichen planus [5], mucosal lichen planus, and lichen planus pigmentosus (LPPig) have been reported. The latter supports a shared pathologic pathway between FFA and lichen planopilaris.

Clinical features of LPPig, an uncommon variant of lichen planus, were first described in 1974 by Bhutani et al. [6] in Indian patients. The association between FFA and LPPig was first described by Dlova in 2013 in African women [7]. Since then, most publications about this association involved dark-skinned patients. Pirmez et al. [8] described the clinical and dermoscopic features of LPPig in 37 patients with FFA, most of them with high phototypes. Romiti et al. [9] reported 2 skin phototype-II cases in a series of 16 mainly darker skin phototype patients. Vaño-Galván et al. [10] published in 2014 one of the largest series of AFF in Caucasian patients. They did not report any LPPig association. This may be an indirect sign that LPPig is not common in light-skinned patients, given that most of the patients were Spanish. We report 5 cases of FFA associated with LPPig in light-skinned Argentine women. 


\section{Case Report/Case Presentation}

We reviewed charts of all FFA patients seen at the Hair Clinic of CEMIC, Buenos Aires Skin, and CIAL from 2008 to 2018 in Buenos Aires and Bahía Blanca (Argentina). Evaluation of 111 patients diagnosed with FFA seen in these clinics revealed 5 patients with the diagnosis of LPPig on the face and neck. FFA was confirmed by scalp biopsy in 2 patients and LPPig by skin biopsy in all of them (Table 1; Fig. 1-3).

\section{Discussion and Conclusion}

All patients were women with light skin phototypes (Fitzpatrick phototypes II, III), aged $45-83$ years. The age at onset of LPPig ranged from 42 to 79 years and of FFA from 42 to 69 years. In 3 patients, LPPig preceded FFA ( 4 months to 4 years). Two patients were premenopausal, and 4 had extra-facial skin involvement (neck) of LPPig. One patient had a maternal history of FFA.

Patient number 4 was diagnosed previously as rosacea because of the facial erythema, as reported by LópezPestaña et al. [11]. Some of these patients are sometimes misdiagnosed with erythematous rosacea, although they never had inflammatory papules or pustules. As published by Gavazzoni Dias et al. [12], 4 women in this series had hyperpigmentation of the upper lid, a clue to the diagnosis of facial LPPig that helps its differentiation from other dermatoses such as melasma.

The clinical variants of LPPig were reticulated in 2 patients and comprised multiple pigmented macules in the others. All the patients had eyebrow alopecia, and facial papules were present in 3 women. Recently, Mervis et al. [13] reported a significant association between Hispanic/ Latino ethnicity, facial papules and premenopausal status, which may portend susceptibility to a more severe disease.

Although it was not systematically recorded in our series, dermoscopy is a great tool to help in the diagnosis of LLPig, as described by Pirmez and other authors [8]. Our patients were treated with the reported options for FFA, 5-alpha-reductase inhibitors and other drugs borrowed from the treatment of lichen planopilaris. Treatment of LPPig is generally disappointing; our patients received topical clobetasol, tacrolimus, lightening creams, and sunscreens. Two patients developed new facial pigmented lesions in spite of treatment.

The inflammatory phase of the skin lesions that affected our patient in case 4 , although uncommon, should remind us that the presence of inflammatory cells in the early phases of LPPig supports the indication for early treatment to avoid chronic persistent pigmentation [14]. Early intervention with systemic steroids or other agents such as methotrexate can probably help prevent hyperpigmentation, as in our case. Also, because of the hyperpigmentation risk associated with antimalarials, caution should be taken when using these drugs for FFA.

This brief communication highlights the strength of the FFA and LPPig association also in lower Fitzpatrick phototypes. As described by Mervis et al. [13], patients who have worse prognostic factors, such as Latino ethnicity, facial papules, and premenopausal status, may deserve a more aggressive treatment option.

Regarding the association, we can think that both entities are within the lichenoid spectrum. We also wondered which patients can develop skin pigmentation, obviously patients with dark skin and perhaps lower phototypes with high facultative pigmentation capacity.

\section{Statement of Ethics}

All subjects gave their written informed consent to publish their cases (including publication of images).

\section{Disclosure Statement}

The authors have no conflicts of interest to declare.

\section{Funding Sources}

The authors did not receive any funding.

\section{Author Contributions}

All authors contributed equally to this manuscript.

References

1 Harries M, Jimenez F, Izeta A, Hardman J, Parameswara Panicker S, Poblet E, et al. Lichen planopilaris and frontal fibrosing alopecia as model epithelial stem cell diseases. Trends Mol Med. 2018;24(5):435-48.

2 Kossard S. Postmenopausal frontal fibrosing alopecia. Scarring alopecia in a pattern distribution. Arch Dermatol. 1994;130(6):7704.

3 Vañó-Galván S, Rodrigues-Barata AR, Urech $\mathrm{M}$, Jiménez-Gómez $\mathrm{N}$, Saceda-Corralo $\mathrm{D}$, Paoli J, et al. Depression of the frontal veins: a new clinical sign of frontal fibrosing alopecia. Am Acad Dermatol. 2015 Jun;72(6):1087-8. 
4 Donati A, Molina L, Doche I, Valente NS, Romiti R. Facial papules in frontal fibrosing alopecia: evidence of vellus follicle involvement. Arch Dermatol. 2011 Dec;147(12):1424-7.

5 Faulkner CF, Wilson NJ, Jones SK. Frontal fibrosing alopecia associated with cutaneous lichen planus in a premenopausal woman. Australas J Dermatol. 2002 Feb;43(1):65-7.

6 Bhutani LK, Bedi TR, Pandhi RK, Nayak NC. Lichen planus pigmentosus. Dermatologica. 1974;149(1):43-50.

7 Dlova NC. Frontal fibrosing alopecia and lichen planus pigmentosus: is there a link? $\mathrm{Br} J$ Dermatol. 2013;168:439-42.

8 Pirmez R, Duque-Estrada B, Donati A, Campos-do-Carmo G, Valente NS, Romiti R, et al. Clinical and dermoscopic features of lichen planus pigmentosus in 37 patients with frontal fibrosing alopecia. Br J Dermatol. 2016 Dec;175(6):1387-90.
9 Romiti R, Biancardi CF, Anzai A, Munck A, Oliveira Costa CO, Valente NYS. Clinical and histopathological findings of frontal fibrosing alopecia-associated lichen planus pigmentosus. Skin Appendage Disord. 2017;3:59-63.

10 Vañó-Galván S, Molina-Ruiz AM, SerranoFalcón C, Arias-Santiago S, Rodrigues-Barata AR, Garnacho-Saucedo G, et al. Frontal fibrosing alopecia: a multicenter review of 355 patients. J Am Acaddermatol. 2014;70:670-8.

11 López-Pestaña A, Tuneu A, Lobo C, Ormaechea N, Zubizarreta J, Vildosola S, et al. Facial lesions in frontal fibrosing alopecia (FFA): clinicopathological features in a series of 12 cases. J Am Acad Dermatol. 2015 Dec;73(6): 987-6.
12 Gavazzoni Dias MFR, Rezende HD, Lofeu Cury A, Trüeb RM, Vilar E. Hyperpigmented upper eyelid: a clue to the diagnosis of facial lichen planus pigmentosus in a patient with frontal fibrosing alopecia. Skin Appendage Disord. 2018 Oct;4(4):335-8.

13 Mervis JS, Borda LJ, Miteva M. Facial and extrafacial lesions in an ethnically diverse series of 91 patients with frontal fibrosing alopecia followed at a single center. Dermatology. 2018 Nov 22:235(2):112-9.

14 Bhat RM, Mathanda TR, Jayaprakash CS, Dandakeri S. Clinical, histopathologic charactheristic and immunohistochemical findings in lichen planus pigmentosus. Indian J Dermatol. 2017 Nov-Dec:62(6):612-7. 